COMUNICAÇÃO CIENTÍFICA

\title{
CARACTERIZAÇÃO DOS FRUTOS DE GOIABEIRA E SELEÇÃO DE CULTIVARES NA REGIÃO DO SUBMÉDIO SÃO FRANCISCO ${ }^{1}$
}

\author{
MARIA AUXILIADORA COÊLHO DE LIMA², JOSTON SIMÃO DE ASSIS ${ }^{3}$ e \\ LUIZ GONZAGA NETO ${ }^{4}$
}

\begin{abstract}
RESUMO - O estudo visou a caracterizar frutos de goiabeira e selecionar cultivares produzidas na Região do Submédio São Francisco. Os frutos, colhidos na maturidade fisiológica, foram provenientes da Estação Experimental de Bebedouro, pertencente à Embrapa SemiÁrido e avaliados em delineamento experimental inteiramente casualizado com quatro repetições. Os tratamentos compreenderam genótipos de polpa branca (Alabama Safed, Lucknow 49, Banahas, White Selection of Florida e Seleção IPA B 38.1) e vermelha (Paluma, Patillo 2.1, Surubim, Red Selection of Florida e Seleção IPA B 14.3). Avaliaram-se: massa, diâmetro longitudinal (DL), diâmetro transversal (DT), relação DL/DT, espessura da casca, espessura da polpa, sólidos solúveis totais (SST), acidez total titulável (ATT), relação SST/ ATT, $\mathrm{pH}$, açúcares solúveis totais (AST) e vitamina C. Os genótipos de polpa branca, exceto Seleção IPA B 38.1, tiveram maiores massas (acima de 145,0 g). As menores relações DL/DT foram observadas em Banahas $(0,98)$ e Lucknow 49 (1,00), caracterizando frutos redondos. Estas cultivares tiveram também maior espessura da polpa. Os maiores valores de SST foram observados em Paluma (10,4 ${ }^{\circ}$ Brix) e Lucknow 49 (10,9 ${ }^{\circ}$ Brix). A ATT foi baixa na maioria dos genótipos, exceto Surubim e Seleção IPA B 14.3, e o pH variou de 3,72 a 4,22. Em geral, os genótipos de polpa branca tiveram os maiores teores de AST ( 5 a 7 \%) e vitamina C, atingindo $200 \mathrm{mg}$ de ácido ascórbico/100 g.
\end{abstract}

Termos para indexação: características físico-químicas, características químicas e físicas, goiaba de mesa, goiaba para indústria, qualidade.

\section{CHARACTERIZATION OF GUAVA FRUITS AND CULTIVAR SELECTIONS IN THE SUBMÉDIO SÃO FRANCISCO REGION OF BRAZIL}

\begin{abstract}
This study aimed to characterize fruits of guava and to select cultivars produced in the Submédio São Francisco Region. The fruits were produced in the Bebedouro Experimental Station, Embrapa Semi-Árido. They were harvested in the physiological maturity. The experimental design was a completely randomized design with four replications. The treatments were white pulp genotypes (Alabama Safed, Lucknow 49, Banahas, White Selection of Florida and Seleção IPA B 38.1) and red pulp genotypes (Paluma, Patillo 2.1, Surubim, Red Selection of Florida and Seleção IPA B 14.3). They evaluated: weight, longitudinal diameter (DL), transverse diameter (DT), DL/DT ratio, peel and pulp thickness, total soluble solids (TSS), total titrable acidity (TTA), TSS/TTA ratio, $\mathrm{pH}$, total soluble sugars (TSA) and vitamin C. White pulp genotypes had the highest weights (over 145.0 g), except the Seleção IPA B 38.1. The lower DL/DT ratios were observed in Banahas (0.98) and Lucknow 49 (1.00), characterizing round fruits. Also, these cultivars had the largest thickness of the pulp. The highest values of TSS were observed in Paluma (10.4 ${ }^{\circ}$ Brix $)$ and Lucknow 49 (10.9 $\left.{ }^{\circ} \mathrm{Brix}\right)$. ATT was low in most of the genotypes, except in Surubim and Seleção IPA B 14.3, and the pH values varied from 3.72 to 4.22. Most of white pulp genotypes had high AST content (5 at $7 \%$ ) and high vitamin C content (up to $200 \mathrm{mg}$ of acid ascorbic/100 g).
\end{abstract}

Index terms: physico-chemical characteristics, table guava, industry guava, quality.

O Estado de Pernambuco destaca-se como segundo produtor brasileiro de goiaba. No ano de 1995, sua produção representou $24 \%$ do total produzido no Brasil (AGRIANUAL, 1999).

Nas áreas irrigadas do Vale do São Francisco, os cultivos de goiabeira têm se expandido, compreendendo uma área de aproximadamente 4000 ha.

Além da expressividade econômica, segundo Pereira e Martinez Júnior (1986), a goiaba é um dos frutos tropicais e subtropicais de maior valor nutricional. Possui conteúdos de açúcares, ferro, cálcio, fósforo e vitaminas $\mathrm{A}, \mathrm{B}$ e $\mathrm{C}$ superiores à maioria das frutas. Tais características são importantes quando o destino da produção é o consumo in natura, mas podem representar uma redução no uso de aditivos químicos nos frutos processados.

As cultivares de goiabeira destinadas à produção de frutos para consumo in natura devem ter frutos com polpa de coloração preferencialmente branca, de tamanho médio ou

1 (Trabalho 010/2001). Recebido: 09/01/2001. Aceito para publicação: 18/10/2001.

2 Eng $^{\mathrm{a}}$ Agrônoma, estudante de doutorado, bolsista da FUNCAP, Universidade Federal do Ceará, CP 6012, CEP 60541-970, Fortaleza, CE. E-mail: macolima@zipmail.com.br

3 Eng $^{\circ}$ Agrônomo, Dr., Pesquisador, Embrapa Semi-Árido, CP 23, CEP 56300-970,Petrolina, PE.

4 Eng $^{\circ}$ Agrônomo, M.Sc., Pesquisador, Embrapa Semi-Árido. 
grande, ovais, com poucas sementes, firmes e doces (Gonzaga Neto, 1990). As cultivares para fins industriais devem produzir frutos de tamanho médio, redondos, com polpa vermelha, espessa e não muito aquosa, com pouca semente, SST de 8,0 a $12,0^{\circ}$ Brix, pH de 3,8 a 4,3 e acidez entre 0,35 e $0,63 \%$ de ácido cítrico.

$\mathrm{Na}$ Região do Submédio São Francisco, os estudos com goiabeira concentraram-se nos aspectos de produção e adaptação de diferentes genótipos às condições locais. Os estudos de composição química dos frutos limitaram-se a determinações de sólidos solúveis totais e acidez total titulável. Daí, a necessidade de estudos mais detalhados a respeito da qualidade dos frutos produzidos na região.

Os constituintes responsáveis pela qualidade dos frutos recebem a influência direta da cultivar, condições climáticas, solo, tratos culturais e estádios de maturação (Hulme, 1970). Não há, portanto, possibilidade de extrapolar os resultados de uma região para a outra, o que implica a necessidade de pesquisas de âmbito regional para o conhecimento da qualidade dos frutos (Esteves e Carvalho, 1982).

O objetivo deste estudo foi caracterizar os frutos de cultivares e seleções de goiabeira introduzidas na Região do Submédio São Francisco através de determinações físicas, físicoquímicas e químicas.

Os frutos, colhidos na maturidade fisiológica, foram provenientes da coleção de genótipos da Estação Experimental de Bebedouro da Embrapa Semi-Árido, em Petrolina-PE. O clima da região é do tipo BSh'W, segundo classificação de Köppen. As plantas foram submetidas a irrigação por sulco, com aplicação de 150 litros de água por planta, a cada dois dias, na fase de crescimento dos frutos.

O trabalho foi conduzido no Laboratório de Fisiologia Vegetal da Embrapa Semi-Árido, em um delineamento experimental inteiramente casualizado, com quatro repetições. Os tratamentos compreenderam cinco cultivares e cinco seleções de goiabeira, incluindo genótipos de polpa branca (Alabama Safed, Lucknow 49, Banahas, White Selection of Florida e Seleção IPA B 38.1) e vermelha (Paluma, Patillo 2.1, Surubim, Red Selection of Florida e Seleção IPA B 14.3). Cada unidade experimental foi representada por três frutos selecionados de uma amostra homogênea de 30 frutos selecionados entre aqueles obtidos na primeira colheita $\mathrm{e}$ avaliados individualmente em relação às características físicas e, depois, homogeneizados, constituindo uma amostra composta para as demais determinações.

As características avaliadas foram: a) massa (g): obtida em balança semi-analítica; b) diâmetros longitudinal -DL-e transversal -DT- e espessuras da casca e da polpa $(\mathrm{cm})$ : determinados através de paquímetro; c) relação DL/DT; d) sólidos solúveis totais -SST- $\left({ }^{\circ}\right.$ Brix): segundo recomendação da AOAC (1992); e) acidez total titulável -ATT- (\% de ácido cítrico): por titulação com NaOH 0,1N, conforme recomendação do IAL (1985); f) relação SST/ATT; g) pH: obtido conforme AOAC (1992); h) açúcares solúveis totais -AST- (\%): obtido usando 9,10-dihidro9-oxoantracena, segundo método descrito por Dische (1962); i) vitamina C (mg de ácido ascórbico/100 mL): determinado conforme IAL (1985).

Foram realizadas análises de variância para as variáveis estudadas, utilizando-se do teste de Tukey, a 5\% de probabilidade, para a comparação de médias.

A massa dos frutos variou de 90,8 a 244,5 g/fruto (Tabela
1). Considerando que os frutos destinados ao processamento industrial devem ter tamanho médio (massa superior a $100 \mathrm{~g}$ ), segundo Lima et al.(1999), e que aqueles destinados ao consumo in natura devem ser preferencialmente de médios a grandes, nesta ordem, Alabama Safed, Lucknow 49, White Selection of Florida e Banahas confirmam suas características de goiaba para mesa.

Segundo Gonzaga Neto et al. (1987), a massa média do fruto é uma característica importante, uma vez que, em geral, os frutos de maior massa são também os de maior tamanho, e estes, por sua vez, são mais atrativos ao consumidor.

Os valores observados em White Selection of Florida e Seleção IPA B 38.1 superaram os valores registrados por Gonzaga Neto et al. (1987) com os mesmos genótipos, na Região do Vale do Rio Moxotó. Por outro lado, Seleção IPA B 14.3, Surubim e Red Selecion of Florida tiveram massas de 90,8; 93,8 e 109,8 g, respectivamente, as quais foram levemente inferiores às obtidas por Gonzaga Neto et al. (1986), nas condições do Vale do Rio Moxotó. Estas diferenças podem estar relacionadas com as características climáticas da região, do tipo de solo e de cultivo, fatores importantes que influenciam na qualidade dos frutos (Hulme, 1970). Estes genótipos tiveram massas estatisticamente iguais entre si e aos demais genótipos de polpa vermelha. Os genótipos de polpa branca, exceto Seleção IPA B 38.1, destacaramse pela maior massa dos frutos.

Os valores de DL variaram de 5,84 a 7,60 cm (Tabela 1). Além de apresentar frutos com maior massa, a cultivar Banahas apresentou também o maior DL; entretanto, este comportamento não foi observado em relação a White Selection of Florida que também apresentou um dos maiores valores para DL.

A variação do diâmetro transversal dos frutos foi de 5,30 a 7,79 cm (Tabela 1), sendo resultante das diferenças na massa, uma vez que aqueles de menor massa tiveram DT também menor. Assim, os valores de DT de Seleção IPA B 14.3, Surubim, Patillo 2.1, Paluma, Seleção IPA B 38.1 e Red Selection of Florida não diferiram estatisticamente entre si e foram inferiores aos demais.

A análise isolada das variáveis DL e DT tem pouca importância para a caracterização dos frutos dos genótipos de goiabeira. Entretanto, a relação DL/DT é bastante representativa. Segundo Gerhardt et al. (1997), esta relação indica o formato do fruto e quanto mais próxima de 1 , mais redondo.

A relação DL/DT dos frutos variou de 0,98 a 1,25 (Tabela 1). As menores relações, 0,98 a 1,02 , foram obtidas, respectivamente, nas cultivares Banahas, Lucknow 49, Alabama Safed e R. S. Florida, caracterizando o formato redondo dos frutos. Sharma e Singh (1981), entretanto, caracterizaram o formato dos frutos da cultivar Lucknow 49 como sendo globular oval, ressaltando que podem ocorrer variações, dependendo das condições agroclimáticas e práticas culturais.

Os genótipos Surubim, Paluma, Seleção IPA B 14.3 e Patillo 2.1, por sua vez, tiveram relações DL/DT superiores a 1,1, indicando frutos de formato alongado. Já os frutos de W. S. Florida e Seleção IPA B 38.1, com relação DL/DT de 1,05 e 1,06, podem ser considerados como globular ovais.

Os valores de espessura da casca variaram de 0,10 a 0,36 $\mathrm{cm}$ (Tabela 1). Os genótipos de polpa vermelha apresentaram casca com espessura inferior aos de coloração branca. A maior espessura de casca foi observada na cultivar Alabama Safed, sendo 3,6 vezes mais espessa que Paluma. 
A espessura da polpa variou de 0,60 a $1,30 \mathrm{~cm}$, conforme Tabela. Entre os genótipos de maior espessura da polpa, destacaram-se as cultivares Paluma, Lucknow 49 e Banahas. Para a indústria de sucos, os frutos de Paluma apresentaram maior aproveitamento (Silva Júnior et al., 1999). As outras duas variedades, de polpa branca, apresentaram características que podem viabilizá-las para consumo ao natural do fruto.

As cultivares e seleções de goiabeira tiveram teor de SST variando de 7,2 a 10,9 ${ }^{\circ}$ Brix (Tabela 1). Paluma, Lucknow 49 e Banahas destacaram-se pelos valores mais elevados. No entanto, estes valores foram inferiores aos observados por Gonzaga Neto et al. (1986). Os autores registraram, em Seleção IPA B 14, Seleção IPA B 38, Surubim e Red Selection of Florida, teor de SST de 12; 14; 13 e $10^{\circ}$ Brix, respectivamente.

Altos teores de SST são desejáveis tanto para frutos destinados ao consumo in natura quanto para a indústria. No último caso, segundo Gonzaga Neto et al. (1986), o custo do processamento é menor.

A ATT variou de 0,40 a 1,04\% de ácido cítrico (Tabela 1). Os maiores valores foram observados na Seleção IPA B 14.3 e na seleção Surubim. Segundo Nascimento et al. (1998), uma acidez elevada é importante para o processamento, pois diminui a adição de acidificantes artificiais. No entanto, não é um fator limitante para a escolha do genótipo se outras características forem satisfatórias (Lima et al., 1999).

Baixos teores em ácidos, por sua vez, como obtido nos demais genótipos, especialmente Alabama Safed, são uma característica desejável quando o objetivo é o consumo in natura (Paiva et al., 1997).

As características SST e ATT, isoladamente, podem representar um falso indicativo do sabor dos frutos. Já a relação SST/ATT é considerada uma das formas mais práticas para este fim (Nascimento et al., 1998), principalmente quando o produto se destina ao consumo in natura (Manica et al., 1998).

Os valores mais altos SST/ATT foram observados nas cultivares de polpa branca Alabama Safed $(19,82)$ e Lucknow 49 $(20,08)$, conforme a Tabela 1. A menor relação $(9,02)$ foi observada na seleção Surubim.

Os valores de $\mathrm{pH}$ obtidos, variando de 3,72 a 4,22 (Tabela1), são considerados altos. Segundo Manica et al. (1998), valores de $\mathrm{pH}$ superiores a 3,5 indicam a necessidade de se adicionar ácidos orgânicos comestíveis no processamento dos frutos, visando a uma melhor qualidade do produto final industrializado. No entanto, entre as cultivares e seleções estudadas, aquelas de polpa vermelha, preferidas para a indústria, apresentaram os mais baixos valores de $\mathrm{pH}$, principalmente a Surubim.

O teor de AST variou de 3,03 a 7,07 \% (Tabela 1). Dos genótipos estudados, apenas a Seleção IPA B 14.3 teve teor de AST fora da faixa de 3,3 a $10,0 \%$ da massa fresca total, considerada por Chan Junior \& Kwork (1976) como representativa do nível de açúcares da goiaba. Os genótipos de polpa branca tiveram os maiores teores de AST. A cultivar Paluma teve o maior percentual de AST, entre as cultivares e seleções de polpa vermelha, embora não tenha diferido estatisticamente de Patillo 2.1, Surubim, Red Selection of Florida, Seleção IPA B 38.1 e Alabama Safed, sendo as duas últimas de polpa branca.

$\mathrm{O}$ teor de vitamina $\mathrm{C}$, característica especialmente importante nos frutos destinados ao consumo in natura, teve valores variando de 52,80 a 209,88 mg de ácido ascórbico/100 g (Tabela 1). Os genótipos de polpa branca, com exceção da cultivar Alabama Safed, tiveram os maiores teores de vitamina C, confirmando sua indicação como goiaba de mesa.

1.Os frutos de polpa branca das variedades Banahas, White Selection of Florida, Lucknow 49 e Alabama Safed destacaram-

TABELA 1 - Características físicas, físico-químicas e químicas de frutos de cultivares e seleções de goiabeiras produzidos na Região do Submédio São Francisco. Embrapa Semi-Árido, Petrolina-PE. 1998.

\begin{tabular}{|c|c|c|c|c|c|c|c|c|c|c|}
\hline & Banahas & W.S. Florida & Lucknow 49 & Alab. Safed & R.S. Florida & Paluma & Sel. IPA B 38.1 & Patillo 2.1 & Surubim & Sel. IPA B 14.3 \\
\hline Massa (g) & $244,5 \mathrm{a}$ & $190,8 \mathrm{~b}$ & $167,8 \mathrm{bc}$ & $145,8 \mathrm{c}$ & $109,8 \mathrm{~d}$ & $104,8 \mathrm{~d}$ & $102 \mathrm{~d}$ & $97,2 \mathrm{~d}$ & $93,8 \mathrm{~d}$ & $90,8 \mathrm{~d}$ \\
\hline $\begin{array}{l}\text { Diâmetro longitudinal } \\
\text {-DL- }(\mathrm{cm})\end{array}$ & $7,60 \mathrm{a}$ & $7,33 \mathrm{ab}$ & $6,82 \mathrm{bc}$ & $6,51 \mathrm{~cd}$ & $5,84 \mathrm{e}$ & 6,29 cde & $6,01 \mathrm{de}$ & $6,72 \mathrm{bc}$ & $6,01 \mathrm{de}$ & 6,33 cde \\
\hline $\begin{array}{l}\text { Diâmetro transversal } \\
\text {-DT- }(\mathrm{cm})\end{array}$ & $7,79 \mathrm{a}$ & $6,99 \mathrm{~b}$ & $6,84 \mathrm{bc}$ & $6,39 \mathrm{c}$ & $5,73 \mathrm{~d}$ & $5,57 \mathrm{~d}$ & $5,67 \mathrm{~d}$ & $5,37 \mathrm{~d}$ & $5,37 \mathrm{~d}$ & $5,3 \mathrm{~d}$ \\
\hline Relação DL/DT & $0,98 \mathrm{a}$ & $1,05 a b c$ & $1,00 \mathrm{a}$ & $1,02 \mathrm{ab}$ & $1,02 \mathrm{ab}$ & $1,13 \mathrm{~cd}$ & $1,06 \mathrm{abc}$ & $1,25 \mathrm{e}$ & $1,12 a b c$ & $1,19 \mathrm{de}$ \\
\hline Espessura da casca $(\mathrm{cm})$ & $0,23 \mathrm{bcd}$ & $0,33 \mathrm{ab}$ & $0,27 \mathrm{abc}$ & $0,36 \mathrm{a}$ & 0,15 cde & $0,10 \mathrm{e}$ & $0,27 \mathrm{abc}$ & 0,14 de & $0,12 \mathrm{de}$ & $0,14 \mathrm{de}$ \\
\hline Espessura da polpa $(\mathrm{cm})$ & $1,30 \mathrm{a}$ & 0,76 bcd & $0,88 \mathrm{~b}$ & $0,68 \mathrm{~cd}$ & $0,79 \mathrm{bc}$ & $0,83 \mathrm{~b}$ & $0,63 \mathrm{~d}$ & $0,60 \mathrm{~d}$ & $0,64 \mathrm{~cd}$ & $0,65 \mathrm{~cd}$ \\
\hline $\begin{array}{l}\text { Sólidos solúveis totais } \\
\left.\text {-SST- ( }{ }^{\circ} \text { Brix }\right)\end{array}$ & $10,2 \mathrm{ab}$ & 8,7 cde & $10,9 \mathrm{a}$ & $7,8 \mathrm{def}$ & $9,2 \mathrm{bc}$ & $10,4 \mathrm{a}$ & 7,7 ef & $7,8 \mathrm{def}$ & $8,9 \mathrm{~cd}$ & $7,2 \mathrm{f}$ \\
\hline $\begin{array}{l}\text { Acidez total titulável } \\
\text {-ATT- (\% ác. cítrico) }\end{array}$ & $0,56 \mathrm{ab}$ & $0,60 \mathrm{ab}$ & $0,54 \mathrm{ab}$ & $0,40 \mathrm{a}$ & $0,58 \mathrm{ab}$ & $0,63 \mathrm{ab}$ & $0,58 \mathrm{ab}$ & $0,63 \mathrm{ab}$ & $1,04 \mathrm{c}$ & $0,71 \mathrm{~b}$ \\
\hline Relação SST/ATT & $18,63 \mathrm{ab}$ & 14,56 abcd & $20,08 \mathrm{a}$ & $19,82 \mathrm{a}$ & $15,82 \mathrm{abc}$ & $17,63 \mathrm{ab}$ & $13,32 \mathrm{bcd}$ & $13,24 \mathrm{bcd}$ & $9,02 \mathrm{~d}$ & $10,24 \mathrm{~cd}$ \\
\hline $\mathrm{pH}$ & $4,16 \mathrm{a}$ & $4,22 \mathrm{a}$ & $4,16 \mathrm{a}$ & $4,12 \mathrm{a}$ & $3,96 \mathrm{~b}$ & $3,88 \mathrm{~b}$ & $3,90 \mathrm{~b}$ & $3,94 \mathrm{~b}$ & $3,72 \mathrm{a}$ & $3,88 \mathrm{~b}$ \\
\hline $\begin{array}{l}\text { Açúcares solúveis totais } \\
\text {-AST- }(\%)\end{array}$ & $7,07 \mathrm{a}$ & $5,90 \mathrm{ab}$ & $7,06 \mathrm{a}$ & $5,50 \mathrm{bc}$ & $3,91 \mathrm{de}$ & $4,61 \mathrm{~cd}$ & $4,52 \mathrm{~cd}$ & $3,63 \mathrm{de}$ & $3,79 \mathrm{de}$ & $3,03 \mathrm{e}$ \\
\hline $\begin{array}{c}\text { Vitamina C } \\
\text { (mg ác. Ascórbico/100mL) }\end{array}$ & $145,22 \mathrm{bc}$ & $209,88 \mathrm{a}$ & $158,40 \mathrm{~b}$ & $52,80 \mathrm{f}$ & 64,68 ef & $89,78 \mathrm{~d}$ & $129,35 \mathrm{c}$ & $95,05 \mathrm{~d}$ & 64,7 ef & 87,12 de \\
\hline
\end{tabular}


se por apresentar massa superior a 145,0 g/fruto, DL e DT superior a 6,5 e $6,3 \mathrm{~cm}$, respectivamente. Estes genótipos e a Seleção IPA B 38.1 produziram frutos redondos, com espessura de casca superior a $0,22 \mathrm{~cm}$.

2.As cultivares Banahas, Lucknow 49 e Paluma apresentam espessura de polpa superior aos demais genótipos estudados. No entanto, não foi possível caracterizar um valor aproximado de espessura de polpa para os grupos de genótipos de cor branca e vermelha, já que algumas cultivares ou seleções de um têm médias estatisticamente iguais ao do outro.

3.As cultivares Banahas, Lucknow 49 e Paluma também tiveram os mais altos teores de SST: 10,2, 10,9 e 10,4 ${ }^{\circ}$ Brix, respectivamente. 4.Os frutos de polpa vermelha tiveram, em geral, ATT maiores que os de polpa branca. Conseqüentemente, os valores de $\mathrm{pH}$ foram menores naqueles frutos.

5.Os mais altos teores de AST foram observados nas cultivares Banahas (7,07 \%) e Lucknow 49 (7,06 \%), que também apresentaram valores elevados de vitamina C: 145,22 e 158,40 mg de ácido ascórbico/100 g, respectivamente. O conteúdo mais alto de vitamina C $(209,88 \mathrm{mg}$ de ácido ascórbico/100 g) foi observado na White Selection of Florida.

6. As cultivares Banahas e Luknow demonstraram potencial para produção de frutos para consumo, enquanto a Paluma e a Red Selection of Florida demonstraram potencial para produção de frutos para consumo e para a indústria, nas áreas irrigadas do Submédio São Francisco.

\section{REFERÊNCIAS BIBLIOGRÁFICAS}

AGRIANUAL. Anuário da agricultura brasileira. FNP Consultoria \& Comércio. M \& S Mendes \& Scotoni, Editora Argos Comunicação, 1999. 521p.

ASSOCIATION ON OFFICIAL AGRICULTURAL CHEMISTS. Official methods of analysis of the Association of the Agricultural Chemists. 11 ${ }^{\mathrm{a}} \mathrm{ed}$. Washington: AOAC, 1992. 1115p.

CHAN JUNIOR, H.T.; KWORK, S.C.M. Identification and determination of sugars in some tropical fruit products. Journal of Food Science, Chicago, v.40, n.2, p.419-420, 1976.

DISCHE, Z. General color reactions. In: WHISTLER, R.L.; WOLFRAM, M.L. Carbohydrate chemistry. New York: Academic Press, 1962.p.477-512.

ESTEVES, M.T. da C.; CARVALHO, V.D. de. Modificações nos teores de amido, açúcares e grau de doçura de seis cultivares de goiabeira (Psidium guajava L.) em diferentes estádios de maturação. Ciência e Prática, Lavras, v.6, n.2, p.208-218, 1982.

GERHARDT, L.B. de A.; MANICA, I.; KIST, H.; SIELER, R.L. Características físico-químicas dos frutos de quatro cultivares e três clones de goiabeira em Porto Lucena, RS. Pesquisa Agropecuária Brasileira, Brasília, v.32, n.2, p.185-192, 1997.

GONZAGA NETO, L. Cultura da goiabeira. Petrolina, PE: EMBRAPA-CPATSA, 1990. 26p. (Circular Técnica, 23).
GONZAGA NETO, L.; ABRAMOF, L.; BEZERRA, J.E.F.; PEDROSA, A.C.; SILVA, H.M. Seleção de cultivares de goiabeira (Psidium guajava L.) para consumo ao natural, na Região do Vale do Rio Moxotó, em Ibibmirim-Pernambuco. Revista Brasileira de Fruticultura, Cruz das Almas, v.9, n,2, p.63-66, 1987.

GONZAGA NETO, L.; PEDROSA, A.C.; ABRAMOF, L.; BEZERRA, J.E.F.; DANTAS, A.P.; SILVA, H.M.; SOUZA, M.M. de. Seleção de cultivares de goiabeira (Psidium guajava L.) para fins industriais, na Região do Vale do Rio Moxotó. Revista Brasileira de Fruticultura, Cruz das Almas, v.8, n..2,p.55-61, 1986.

HULME, A.C. The biochemistry of fruits and their products. New York, Academic Press, 1970, 2v.

INSTITUTO ADOLFO LUTZ. Normas analíticas, métodos químicos e físicos para análise de alimentos. São Paulo, 1985, 371p. v.1.

LIMA, M.A.; DURIGAN, J.F.; PEREIRA, F.M.; FERRAUDO, A.S. Caracterização físico-química dos frutos de 19 genótipos de goiabeira, obtidos na FCAV-UNESP. Revista Brasileira de Fruticultura, Jaboticabal, v.21, n.3, p.252-257, 1999.

MANICA, I.; KIST, H.; MICHELETTO, E.L.; KRAUSE, C.A. Competição entre quatro cultivares e duas seleções de goiabeira. Pesquisa Agropecuária Brasileira, Brasília, v.33, n.8, p.13051313, 1998.

NASCIMENTO, T.B. do; RAMOS, J.D.; MENEZES, J.B. Características físico-químicas do maracujá-amarelo (Passiflora edulis f. flavicarpa Degener) produzido em diferentes épocas. Revista Brasileira de Fruticultura, Cruz das Almas, v.20, n.1, p.33-38, 1998.

PAIVA, M.C.; MANICA, I.; FIORAVANÇO, J.C.; KIST, H. Caracterização química dos frutos de quatro cultivares e duas seleções de goiabeira. Revista Brasileira de Fruticultura, Cruz das Almas, v.19, n.1, p.57-63, 1997.

PEREIRA, F.M.; MARTINEZ JUNIOR, H. Goiabas para industrialização. Jaboticabal: UNESP, 1986. 142p.

SHARMA, A.K.; SINGH, S.N. Physico-chemical characteristics of some guava (Psidium guajava L.) cultivars. Food Farming and Agriculture, v.13, n.11/12, p.191-193, 1981.

SILVA JÚNIOR, J.F. da; TAVARES, J.A.; BEZERRA, J.E.F.; LEDERMAN, I.E.; PEDROSA, A.C.; MELO NETO, M.L. de. Competição de cultivares de goiabeira (Psidium guajava L.) para indústria na Chapada do Araripe, PE. II. Características físicoquímicas do fruto. Revista Brasileira de Fruticultura, Jaboticabal, v.21, n.1, p.1-6, 1999. 